\title{
COMORBIDADES E ESTILO DE VIDA DE IDOSOS COM TUBERCULOSE
}

\author{
Comorbidities and lifestyle of the elderly with tuberculosis \\ Comorbidades y estilo de vida en mayores con tuberculosis
}

Artigo Original

\section{RESUMO}

Objetivo: Analisar as comorbidades e o estilo de vida de idosos com tuberculose. Métodos: Realizou-se um estudo caso-controle com 48 idosos que tiveram tuberculose (TB) e 96 controles vizinhos, nos anos 2008/2009, em Teresina-PI. Coletaram-se informações demográficas, sobre comorbidades, hábitos de vida e dados clínicos através do Sistema de Informação de Agravos de Notificação (SINAN) do município. Utilizou-se o odds ratio (OR) como medida de associação, pelo programa SPSS. Resultados: A média de idade dos casos era de $74( \pm 8,0)$ anos e dos controles, de $73( \pm 9,0)$ anos. Houve maior ocorrência do sexo masculino $(52 \% ; n=25)$ entre os casos $(p=0,039)$. Com relação à moradia, $92 \%(n=44)$ dos casos de TB e $90 \%(n=86)$ dos controles moravam em casa própria, enquanto $75 \%(n=36)$ dos casos e $71 \%(n=68)$ dos controles residiam com duas a cinco pessoas $(p>0,05)$. Nos casos, verificou-se que $52 \%(n=25)$ eram fumantes e $58 \%(n=28)$, etilistas. Entretanto, não houve associação entre os grupos. Apesar de $50 \%(n=24)$ dos casos serem hipertensos e $15 \%$ $(n=7)$ terem diabetes mellitus ou doença cardíaca, não houve associação entre os grupos. Observou-se associação $(\mathrm{p}=0,003)$ entre a ocorrência de novos casos e o contato próximo com portadores de TB. Entre esses casos anteriores, 85,4\% $(n=41)$ tinham a mesma faixa de idade dos casos. Conclusão: Observou-se que fatores como comorbidades, hábitos de vida (tabagismo e alcoolismo) e condições de moradia não apresentaram diferença significativa entre os idosos que tiveram tuberculose e os controles vizinhos na mesma faixa etária.

Descritores: Tuberculose; Idoso; Estudos de Casos e Controles.

\section{ABSTRACT}

Objective: To analyse the comorbidities and lifestyle of elderly people with tuberculosis. Methods: A case-control study was performed with 48 elderly patients who have had tuberculosis (TB) and 96 neighbours as controls, in the years 2008/2009, in Teresina, Piaui. Information on demographics, comorbidities, lifestyle, and clinical data were collected through the Notifiable Diseases Information System (SINAN) in the city. The odds ratio (OR) was used as a measure of association, in SPSS software. Results: The average age of cases was $74( \pm 8.0)$ years, and 73 ( \pm 9.0$)$ years among controls. There was a higher occurrence of males $(52 \% ; n=25)$ among cases $(p=0.039)$. As for housing, 92\% $(n=44)$ of TB cases and $90 \%(n=86)$ of the controls lived in their own home, while $75 \%(n=36)$ of cases, and $71 \%$ $(n=68)$ of controls lived with two to five people $(p>0.05)$. Among cases, it was found that $52 \%$ $(n=25)$ were current smokers, and $58 \%(n=28)$ were alcoholics. However, no association was found between the groups. Although 50\% $(n=24)$ of patients were hypertensive and $15 \%(n=7)$ had diabetes mellitus or heart disease, there was no association between the groups. An association ( $p=0.003)$ was observed between the occurrence of new cases and close contact with people with TB. Conclusion: The study observed that factors like comorbidities, lifestyle (smoking and alcoholism), and housing conditions did not present significant difference between the elderly with tuberculosis and the neighbour controls in the same age range.

Descriptors: Tuberculosis; Elderly; Case-Control Studies.

\author{
Danieli Maria Matias Coêlho ${ }^{(1,2)}$ \\ José Machado Moita Neto ${ }^{(1)}$ \\ Viriato Campelo $^{(1)}$
}

1) Universidade Federal do Piauí - UFPI Teresina (PI) - Brasil

2) Centro de Ensino Unificado de Teresina CEUT - Teresina (PI) - Brasil

Recebido em: $11 / 11 / 2013$ Revisado em: 25/03/2014 Aceito em: 18/06/2014 


\section{RESUMEN}

Objetivo: Analizar las comorbidades y el estilo de vida en mayores con tuberculosis. Métodos: Se realizó un estudio caso-control con 48 mayores que tuvieron tuberculosis (TB) en 2008/2009 y 96 controles vecinos en Teresina-PI. Se recogieron informaciones demográficas, de las comorbidades, hábitos de vida $y$ datos clínicos a través del Sistema de Información de Agravos de Notificación (SINAN) del municipio. Se utilizó el odds ratio (OR) como medida de asociación en el programa SPSS. Resultados: La media de edad de los casos fue de $74( \pm 8,0)$ años y de los controles de $73( \pm 9,0)$ años. Hubo mayor ocurrencia del sexo masculino $(52 \% ; n=25)$ entre los casos $(p=0,039)$. Respecto la vivienda el 92\% $(n=44)$ de los casos de TB y el 90\% $(n=86)$ de los controles vivían en casa propia mientras el $75 \%(n=36)$ de los casos y el $71 \%(n=68)$ de los controles vivían entre dos y cinco personas $(p>0,05)$. En los casos se verifico que el $52 \%(n=25)$ eran fumadores y el $58 \%(n=28)$, eran alcohólicos. Sin embargo, no hubo asociación entre grupos. A pesar de que el 50\% $(n=24)$ de los casos eran hipertensos y el 15\% $(n=7)$ tenían diabetes mellitus o enfermedad cardíaca, no hubo asociación entre grupos. Se observó asociación $(p=0,003)$ entre la ocurrencia de casos nuevos y el contacto próximo con portadores de TB. De los casos anteriores, el 85,4\% $(n=41)$ tenían la misma franja de edad de los casos. Conclusión: Se observó que factores como comorbidades, hábitos de vida (tabaco y alcoholismo) y las condiciones de vivienda no presentaron diferencia significativa entre los mayores que tuvieron tuberculosis y los controles vecinos con la misma franja de edad.

Descriptores: Tuberculosis; Anciano; Estudios de Casos y Controles.

\section{INTRODUÇÃO}

Causada pelo bacilo Mycobacterium tuberculosis, a tuberculose (TB) é considerada um grave problema de saúde pública, contribuindo para isso as desigualdades sociais, o envelhecimento da população, a insuficiência de pesquisas visando ao desenvolvimento de novos tratamentos e vacinas, os movimentos migratórios, as deficiências dos sistemas de saúde e a alta prevalência dos casos de tuberculose multidrogas resistentes ${ }^{(1,2)}$.

O Brasil ocupa atualmente a $16^{\mathrm{a}}$ posição entre os 22 países que concentram cerca de $80 \%$ dos casos de tuberculose do mundo, em números absolutos. Em 2013, o país notificou 71.123 casos novos, correspondendo a um coeficiente de incidência de 35,4/100.000 hab. Considerando-se esse coeficiente, o país ocupa a $22^{\mathrm{a}}$ posição entre esses países. O Ministério da Saúde (MS) definiu a TB como prioridade entre as políticas públicas, e o Programa Nacional de Controle da Tuberculose (PNCT), seguindo as diretrizes da Organização Mundial de Saúde, estabelece metas de diagnosticar $70 \%$ dos casos esperados e curar pelo menos $85 \%$ deles $^{(2-4)}$.

Em conformidade com a literatura, segundo a qual as capitais possuem as mais altas incidências de tuberculose devido à alta densidade demográfica e aos bolsões de pobreza, o município de Teresina apresentava, em 2005, taxa de incidência acima da média nacional, com 50,2 casos/100.000 hab., enquanto o estado apresentava incidência em torno de 36,2/100.000 hab. ${ }^{(5-8)}$.

Somado a esses fatores, a TB está inserida em um contexto epidemiológico mundial de transição demográfica de franco envelhecimento populacional. Isso pode ser observado no Brasil, onde a incidência começa a se deslocar para a faixa etária dos idosos ${ }^{(9,10)}$. De acordo com as projeções estatísticas da OMS, o grupo de idosos no país deverá ter aumentado em quinze vezes no período de 1950 a $2025^{(11)}$.

Entre os fatores que favorecem a infecção tuberculosa no idoso, devem-se citar as comorbidades, a imunodepressão, o alcoolismo, o tabagismo, as drogas e a má alimentação. As doenças associadas destacam-se nos idosos da América Latina, pela situação econômica e pela dificuldade de acesso aos serviços de saúde ${ }^{(10)}$.

Diante do exposto, este estudo teve como objetivo analisar as comorbidades e o estilo de vida de idosos com tuberculose.

\section{MÉTODOS}

Realizou-se um estudo caso-controle para análise das comorbidades e do estilo de vida dos casos novos de TB em idosos $^{(11)}$, notificados nos anos de 2008 e 2009.

Este estudo foi conduzido em Teresina, capital do estado do Piauí, localizada no centro-norte do estado e meio-norte do Nordeste brasileiro. A população de Teresina, no ano de 2006, era de 801.972 habitantes, dos quais $49.816(6,2 \%)$ tinham 60 anos de idade ou mais. Em 2009, a população era de 802.565 habitantes, dos quais 54.349 (6,8\%) eram idosos $^{(12,13)}$

Em 2008 e 2009, notificaram-se, respectivamente, 61 e 64 casos novos de TB em idosos e residentes em Teresina, num total de 125 casos. Destes, excluíram-se 77 casos da pesquisa, pois 7 tiveram óbito por TB, 25 tiveram óbito por outros motivos, 35 não foram localizados, 5 eram duplicidade, 2 não consentiram com a entrevista e 3 faziam parte do teste piloto. Assim, participaram 48 casos de TB, dos quais 23 foram notificados no Sistema de Informação de Agravos de Notificação (SINAN) em 2008 e 25, em 2009.

A identificação dos casos ocorreu a partir do SINAN, versão 4.0, por meio de tabelas do programa TABWIN, versão 3.2 , contendo o nome do paciente, número do 
SINAN, Unidade de Saúde notificadora, endereço, nome da mãe e data de nascimento.

Para o total de 48 casos, participaram 96 controles, na mesma faixa etária, residentes próximos aos casos (vizinhos), sendo dois controles vizinhos para cada caso. Em situação de recusa, escolheu-se outro controle (vizinho residente na mesma área do caso) para participação.

Após a seleção, foi aplicado o formulário semiestruturado, que constava de questões abertas e fechadas, para o levantamento de informações demográficas (idade, sexo, condições de moradia e renda familiar), sobre comorbidades (diabetes mellitus, hipertensão arterial sistêmica, doença cardíaca, doença renal, câncer, reumatismo, asma, AIDS e silicose), hábitos de vida (etilismo e tabagismo e uso de drogas ilícitas) e dados clínicos (história de contato e tratamento anterior para TB).

Considerou-se etilismo $\mathrm{o}$ ato de ingerir bebida alcoólica, categorizado em etilismo social (no máximo nos fins de semana e/ou comemorações) ou excesso (beber todos os dias ou quando começa a beber não consegue parar); e tabagismo, o hábito de fumar. Os dois hábitos, bem como a utilização de drogas ilícitas, foram investigados quanto a serem atuais (há pelo menos seis meses da aplicação do formulário) ou passados (há mais de seis meses).

Realizou-se um teste piloto para analisar a viabilidade dos formulários, através da verificação dos termos, forma, ordem e clareza das questões e tempo de aplicação, sendo realizados os ajustes necessários para se proceder a coleta de dados.

No caso de pacientes portadores de transtornos mentais, não sendo possível a aplicação do formulário, este foi aplicado ao responsável/cuidador desse participante.

Os dados foram tabulados utilizando-se as bases populacionais por ano, sexo e faixa etária, obtidas do site do Datasus - $\mathrm{MS}^{(12)}$. Descreveram-se as variáveis quantitativas por seus valores médios e respectivos desvios-padrão. As variáveis categóricas foram descritas por seus valores de frequência.

Para analisar a significância das diferenças de médias encontradas, realizou-se o teste " $\mathrm{t}$ " para amostras independentes. Aplicou-se o teste $\mathrm{X}^{2}$ (qui-quadrado) para verificar associação entre variáveis categóricas. Utilizouse o odds ratio (OR), ou razão de chance, como medida de associação das diversas exposições entre os grupos dos casos e dos controles. Adotou-se o nível de significância de $5 \%(\mathrm{p}<0,05)$ e o intervalo de confiança de 95\% (IC95\%). Todos os procedimentos estatísticos foram executados no programa Statistical Package for the Social Sciences (SPSS), versão 15.0 for Windows.

A pesquisa foi autorizada pela Comissão de Ética em Pesquisa da Fundação Municipal de Saúde (FMS) (Parecer $\left.n^{0} 458 / 2010\right)$ e pelo Comitê de Ética em Pesquisa da Universidade Federal do Piauí (Parecer n ${ }^{\circ}$ 0439.0.045.00010), em cumprimento à Resolução $\mathrm{n}^{\circ} 466 / 12$ do Conselho Nacional de Saúde ${ }^{(14)}$.

\section{RESULTADOS}

Nos anos de 2008 e 2009, foram notificados, respectivamente, 61 e 64 casos novos de TB em idosos. Desses, 48 pacientes foram localizados, entrevistados e comparados a 96 controles, ou seja, vizinhos na mesma faixa etária que não tiveram a doença. A idade dos casos variou de 61 a 91 anos, com média de $74( \pm 8,0)$ anos; nos controles, variou de 60 a 93 anos, com média de $73( \pm 9,0)$ anos. Com relação ao sexo, através do teste qui-quadrado, verificou-se uma associação entre gênero e tuberculose no idoso $(\mathrm{p}=0,039)$. A distribuição do sexo masculino $(52 \%$; $\mathrm{n}=25)$ entre os casos de TB foi maior que o feminino $(48 \%$ / $\mathrm{n}=23$ ).

Quanto às condições socioeconômicas, com relação à moradia, $92 \%(\mathrm{n}=44)$ dos casos de TB moram em casa própria, $94 \%(\mathrm{n}=45)$ das casas têm paredes de tijolo com reboco de cimento e $80 \%(\mathrm{n}=38)$ das casas têm piso com azulejo. Percentuais próximos foram encontrados entre os controles, com 90\% ( $\mathrm{n}=86)$ em casa própria, 97\% $(\mathrm{n}=93)$ das casas com parede de tijolo e $88 \%(\mathrm{n}=84)$ com piso de azulejo.

Ainda com relação às condições de moradia, tanto os casos como os controles apresentaram média de 7 cômodos e 4 janelas por residência, assim como a maioria dos casos de TB $(75 \% ; n=36)$ e dos controles $(71 \% ; n=68)$ morava em domicílios com duas a cinco pessoas, com média de 4 pessoas por residência, onde dormiam em média 2 pessoas por quarto, sem diferença estatisticamente significativa nas variáveis acima mencionadas, após o teste " $\mathrm{t}$ " $(\mathrm{p}>0,05)$.

Quanto à renda familiar, $41,7 \%(\mathrm{n}=20)$ dos casos apresentaram renda maior que três salários mínimos; entre os controles, esse percentual foi de somente 19,8\% ( $\mathrm{n}=19)$. Ao analisar essa variável nos casos, verificou-se que a ocorrência de tuberculose está associada ao aumento da renda familiar $(\mathrm{p}=0,05)$.

Quando investigadas as comorbidades entre os casos e controles entrevistados, apesar de $15 \%(\mathrm{n}=7)$ dos casos de TB terem apresentado diabetes mellitus (DM), 50\% $(\mathrm{n}=24)$ possuírem hipertensão arterial sistêmica (HAS) e $15 \%(n=7)$, doença cardíaca, não houve associação entre os casos e os controles em relação a essas comorbidades, assim como nenhum caso de TB investigado apresentava Aids ou silicose e apenas $4 \%(\mathrm{n}=2)$ apresentaram doença renal, $8 \%(\mathrm{n}=4)$ tinham asma e $2 \%(\mathrm{n}=1)$, câncer e reumatismo (Tabela I). 
Tabela I - Análise das comorbidades e hábitos de vida entre os grupos dos casos novos de idosos notificados com tuberculose em 2008 e 2009 e dos controles. Teresina-PI, 2008-2009.

\begin{tabular}{lcccc}
\hline $\begin{array}{l}\text { Comorbidades/ } \\
\text { Hábitos de Vida }\end{array}$ & Categorias & Casos & Controles & $\begin{array}{c}\text { Odds Ratio } \\
\text { (IC 95\%) }\end{array}$ \\
\hline Hipertensão arterial & Sim & 24 & 57 & 0,68 \\
& Não & 24 & 39 & $(0,34-1,37)$ \\
Diabetes mellitus & Sim & 7 & 14 & 1,00 \\
& Não & 41 & 82 & $(0,38-2,67)$ \\
Doença cardíaca & Sim & 7 & 20 & 0,65 \\
& Não & 41 & 76 & $(0,25-1,66)$ \\
Tabagismo & Sim & 32 & 49 & 0,52 \\
& Não & 16 & 47 & $(0,25-1,07)$ \\
Etilismo social & Sim & 28 & 46 & 0,66 \\
& Não & 20 & 50 & $0,33-1,32$ \\
\hline
\end{tabular}

*IC 95\% - Intervalo de confiança 95\%.

Pesquisando-se os hábitos de vida, apesar de $52 \%$ $(\mathrm{n}=25)$ dos casos de TB terem fumado no passado (há mais de 6 meses), $15 \%(\mathrm{n}=7)$ fumarem atualmente e da alta frequência do etilismo social, com $58 \%$ dos pacientes $(\mathrm{n}=28)$ tendo feito uso de bebida alcoólica no passado ou beberem atualmente, não houve associação entre os grupos estudados (Tabela I).

Ainda sobre as comorbidades e os hábitos de vida, utilizando-se o odds ratio (OR) como medida de associação das diversas exposições entre os grupos dos casos e dos controles, observou-se que, entre os idosos participantes, não houve chance de adoecer de tubérculos em relação à hipertensão arterial, diabetes mellitus, doença cardíaca, tabagismo e etilismo social, conforme mostra a Tabela I.

Ao pesquisar se outras pessoas da família ou que residem na casa já tiverem TB, 3 entrevistados não souberam responder. Entre os que souberam dizer, 58,3\% $(\mathrm{n}=28)$ dos casos tiveram contato com pessoas na casa ou na família que tiveram tuberculose, e em $73 \%(n=70)$ dos controles não houve contato com outras pessoas portadoras da doença. Observou-se associação $(\mathrm{p}=0,003)$ entre a ocorrência de novos casos e o contato próximo com portadores de TB. Entre esses casos anteriores, $85,4 \%(n=41)$ têm a mesma faixa de idade dos casos.

Apenas 6\% $(\mathrm{n}=3)$ dos casos tiveram TB no passado: um $(n=1)$ há 19 anos, outro há 37 anos e outro há 47 anos. Todos relataram ter feito tratamento completo à época.

\section{DISCUSSÃO}

Quanto aos idosos notificados em 2008 e 2009, entrevistados e comparados com controles vizinhos na mesma faixa etária, observou-se que, em relação ao sexo, o maior percentual de homens também foi observado em outro estudo de caso-controle, no Rio de Janeiro ${ }^{(15)}$.

De forma similar, estudo desenvolvido no estado de Minas Gerais, que buscou analisar o perfil de casos de tuberculose no estado entre 2002 e 2009 através do SINAN, encontrou predominância em idosos acima de 65 anos, principalmente no sexo masculino, em uma proporção de $2: 1^{(16)}$. A maioria masculina pode estar associada à maior exposição ao Mycobacterium tuberculosis, por esta coorte ter tido maior circulação social no período em que ainda eram jovens ${ }^{(15,17)}$.

Comparando-se casos e controles idosos em relação às comorbidades, observou-se que não houve diferença significativa. Um estudo realizado na Índia mostrou maior prevalência de condições comórbidas, como diabetes mellitus, doenças cardiovasculares e doença pulmonar obstrutiva crônica, em idosos com TB, o que pode ser explicado pelas alterações fisiológicas próprias do envelhecimento ${ }^{(18)}$. Estudo desenvolvido em idosos com tuberculose no Rio de Janeiro ${ }^{(15)}$ aponta que, apesar da elevada proporção de doenças associadas ao grupo de idosos comparados ao grupo de jovens com TB, é possível se tratar de um achado confundidor, visto que tais doenças estão presentes muito frequentemente em qualquer população idosa. Assim, comparando idosos que tiveram a doença com idosos sem TB, observou-se que o desenvolvimento dela pode não estar relacionado à presença de comorbidades, mas ao fato de que aproximadamente $90 \%$ dos casos de tuberculose em idosos têm como causa a reativação da infecção primária ${ }^{(19)}$.

Apesar de não ter encontrado diferença estatisticamente significativa entre casos e controles, em relação ao fumo e ao uso de álcool, observa-se que estes são fatores apontados, 
entre outros, como responsáveis por uma maior morbidade e letalidade da tuberculose no idoso ${ }^{(10)}$.

Os idosos de hoje são de coortes nascidas na década de 1940, tendo sido expostos ao bacilo de Koch durante suas infâncias, quando a doença tinha alta prevalência e os esquemas de tratamento eram menos eficazes. Por esse motivo, estima-se que um elevado percentual dos idosos tenha sido infectado. Essa população, albergando o Mycobacterium tuberculosis e com as deficiências próprias do processo de envelhecimento, pode vir a desenvolver a tuberculose ${ }^{(19)}$.

Também corroborando com o fato de a TB no idoso estar associada às questões do próprio envelhecimento, em que a imunidade celular do indivíduo, considerada a principal responsável pelo combate à infecção pelo bacilo, está diminuída, é que no estudo não foram encontradas diferenças significativas entre casos e controles quanto ao tipo e às condições de moradia e ao número de pessoas por residência e por quarto, apesar de já ter sido demonstrada a elevada taxa de transmissão da doença em ambientes fechados nos países em desenvolvimento ou desenvolvidos ${ }^{(21,20)}$.

Observou-se também que, com relação à renda familiar, os casos apresentaram melhor renda que os controles; assim como em outro estudo caso-controle, em que observaram baixa escolaridade em contradição à melhor renda familiar ${ }^{(9)}$. Uma reflexão sobre o contexto em que se insere a população estudada sugere que talvez isso possa ser explicado pelo fato de os idosos terem filhos com renda própria, ajudando os pais, ou pelo fato de o idoso ter acesso à política de previdência social.

Aproximadamente metade dos casos entrevistados teve contato com pessoas que já tiveram tuberculose (pessoas da casa ou da família), porém, observou-se que essas pessoas tinham a mesma faixa etária dos idosos atualmente entrevistados, e a maioria delas teve a doença há cerca de 24 anos, indicando que esse contato não aconteceu recentemente, que a TB nos casos estudados não é uma infecção recente, e que isso pode ter contribuído para que, na atualidade, tenha ocorrido uma reativação de uma infecção endógena, adquirida no passado.

Em um estudo realizado na Sérvia, observou-se que o fator de risco mais importante para o desenvolvimento da infecção tuberculosa no idoso é considerada a reativação da doença que tinha sido adquirida anteriormente ${ }^{(22)}$. Outro estudo, realizado no Rio de Janeiro, mostra que o desenvolvimento da TB pode ter sido preferencialmente endógeno, pois $70 \%$ dos idosos não tinham antecedentes da doença ${ }^{(15)}$.

Por fim, o presente estudo ressalta a importância de estratégias específicas para prevenir e controlar a tuberculose nos idosos, os quais devem ser considerados grupo vulnerável, definindo-se como uma prioridade entre as políticas públicas.

\section{CONCLUSÃO}

Observou-se que fatores como hábitos de vida (tabagismo e alcoolismo), comorbidades e condições de moradia não apresentaram diferença significativa entre os idosos que tiveram tuberculose e os controles vizinhos na mesma faixa etária.

\section{REFERÊNCIAS}

1. Barreira D, Grangeiro A. Avaliação das estratégias de controle da tuberculose no Brasil. Rev Saúde Pública 2007;41(Supl 1):4-8.

2. Ministério da Saúde (BR), Secretaria de Vigilância em Saúde, Departamento de Vigilância Epidemiológica. Manual de recomendações para o controle da tuberculose no Brasil. Brasília: Ministério da Saúde, 2011.

3. Ministério da Saúde (BR), Secretaria de Vigilância em Saúde. O controle da tuberculose no Brasil: avanços, inovações e desafios. Bol Epidemiológico[periódico na Internet]. 2014 [acesso em 2014 Mai 27];44(2). Disponível em: http://www.vigilanciaemsaude.ba.gov. br/sites/default/files/Boletim-Tuberculose-2014.pdf

4. Ministério da Saúde (BR), Secretaria de Vigilância em Saúde.: Especial tuberculose. Bol Epidemiológico [periódico na Internet]. 2012 [acesso em 2014 Mai 28];43. Disponível em: http://www.saude.rs.gov. br/upload/1337634001_Tuberculose-Boletim\%20 Epidemio.pdf

5. Ministério da Saúde (BR), DATASUS, Cadastro Nacional dos Estabelecimentos de Saúde do Brasil - CNES. Informações de saúde: rede assistencial: CNES - Equipes de Saúde - Piauí [Internet]. Brasília: Ministério da Saúde, 2011 [acesso em 2010 Abr 21]. Disponível em: http://tabnet.datasus.gov.br/cgi/tabcgi. exe?cnes/cnv/equipepi.def

6. Xavier MIM, Barreto ML. Tuberculose na Cidade de Salvador, Bahia, Brasil: O Perfil na Década de 1990. Cad Saúde Pública. 2007;23(2):445-53.

7. Montechi LN, Coêlho DMM, Oliveira CAR, Campelo V. Distribuição espacial da tuberculose em Teresina, Piauí, de 2005 a 2007. Epidemiol Serv Saúde. 2013;22(3):475-82. 
8. Coêlho DMM, Viana, RL, Madeira CA, Ferreira LOC, Campelo V. Perfil epidemiológico da tuberculose no Município de Teresina-PI, no período de 1999 a 2005. Epidemiol Serv Saúde. 2010;19(1):33-42.

9. Cavalcanti ZR, Albuquerque MFPM, Campello ARL, Ximenes R, Montarroyos U, Verçosa MKA. Características da tuberculose em idosos no Recife (PE): contribuição para o programa de controle. J Bras Pneumol. 2006;32(6):535-43.

10. Vendramini SHF, Villa TCS, Gonzales RIC, Monroe AA. Tuberculose no idoso: análise do conceito. Rev Latinoam Enferm. 2003;11(1):96-103.

11. Ministério da Saúde (BR). Portaria ${ }^{\circ} 2.528$, de 19 de outubro de 2006. Aprova a Política Nacional de Saúde da Pessoa Idosa. Brasília: Diário Oficial da União, Brasília, 2006 Out 19.

12. Ministério da Saúde (BR), DATASUS. Informações de saúde: informações demográficas e socioeconômicas: População residente - Piauí [Internet]. Brasília: Ministério da Saúde. [acesso em 2010 Mai 2]. Disponível em: http://www.datasus.gov.br/

13. Prefeitura Municipal de Teresina (PMT), Secretaria Municipal de Desenvolvimento Econômico e Turismo - SEMDEC. Perfil de Teresina: Econômico, social, físico e demográfico. Teresina: Prefeitura Municipal de Teresina; 2010.

14. Ministério da Saúde (BR), Conselho Nacional de Saúde (CNS). Resolução n ${ }^{\circ} .466$ de 12 de dezembro de 2012. Trata de pesquisas envolvendo seres humanos e atualiza a resolução $n^{\circ} 196$. Diário Oficial da União, Brasília, 2012 Dez 13.

15. Cantalice Filho JP, Santa' anna CC, Bóia MN. Aspectos clínicos da tuberculose pulmonar em idosos atendidos em hospital universitário do Rio de Janeiro, RJ, Brasil. J Bras Pneumol. 2007;33(6):699-706.
16. Augusto CJ, Carvalho WS, Gonçalves AD, Ceccato MGB, Miranda SS. Characteristics of tuberculosis in the state of Minas Gerais, Brazil: 2002-2009. J Bras Pneumol. 2013;39(3):357-64.

17. Cullinan P, Meredith SK. Deaths in adults with notified pulmonary tuberculosis 1983-5. Thorax. 1991;46(5):347-50.

18. Gupta D, Singh N, Kumar R, Jindal SK. Manifestations of pulmonary tuberculosis in the Elderly: A prospective observational study from North India. Indian J Chest Dis Allied Sci. 2008;50(3):263-67.

19. Schaaf HS, Collins A, Bekker A, Davies PDO. Tuberculosis at extremes of age. Respirol. 2010;15(5):747-63.

20. Chaimowicz F. Transição etária de incidência e mortalidade por tuberculose no Brasil. Rev Saúde Pública. 2001;35(1):81-7.

21. Conde MB, Melo FAF, Marques AMC, Cardoso NC, Pinheiro VGF, Dalcin PTR, et al. III Diretrizes para Tuberculose da Sociedade Brasileira de Pneumologia e Tisiologia. J Bras Pneumol. 2009;35(10):1018-48.

22. Pesut DP, Gledovic ZB, Grgurevic AD, NagomiObradovic LM, Adzic TN. Tuberculosis incidence in elderly in Serbia: Key trends in socioeconomic transition. Croat Med J. 2008;49(6):807-12.

\section{Endereço para correspondência:}

Danieli Maria Matias Coêlho

Rua Farmacêutico João Carvalho, 4962

Bairro: Santa Isabel

CEP 64.053-150 - Teresina - PI - Brasil

E-mail: danielibrisa@hotmail.com 